\title{
Drug efficacy by direct and adjusted indirect comparison to placebo: An illustration by Mycobacterium avium complex prophylaxis in HIV
}

\author{
Jennifer Chu ${ }^{1 *}$, Caroline E Sloan ${ }^{1}$, Kenneth A Freedberg ${ }^{1,2,3}$, Yazdan Yazdanpanah5, Elena Losina ${ }^{3,4}$
}

\begin{abstract}
Background: Our goal was to illustrate a method for making indirect treatment comparisons in the absence of head-to-head trials, by portraying the derivation of published efficacies for prophylaxis regimens of HIV-related opportunistic infections.

Results: We identified published results of randomized controlled trials from the United States in which HIV-infected patients received rifabutin, azithromycin, clarithromycin, or placebo for prophylaxis against Mycobacterium avium complex (MAC). We extracted the number of subjects, follow-up time, primary MAC events, mean CD4 count, and proportion of subjects on mono or dual antiretroviral therapy (ART) from each study. We derived the efficacy of each drug using adjusted indirect comparisons and, when possible, by direct comparisons. Five articles satisfied our inclusion criteria. Using direct comparison, we estimated the efficacies of rifabutin, clarithromycin, and azithromycin compared to placebo to be 53\% (95\% Cl, 48-61\%), 66\% (95\% Cl, 61-74\%), and 66\% (95\% Cl, 60-81\%), respectively. Using adjusted indirect calculations, the efficacy of rifabutin compared to placebo ranged from $41 \%$ to $44 \%$. The adjusted indirect efficacies of clarithromycin and azithromycin were estimated to be $73 \%$ and $72 \%$, respectively.

Conclusions: Accurate estimates of specific drug dosages as compared to placebo are important for policy and implementation research. This study illustrates a simple method of adjusting for differences in study populations by using indirect comparisons in the absence of head-to-head HIV clinical trials.
\end{abstract}

\section{Background}

Cost-effectiveness analyses are frequently used to guide health policy decisions, particularly in HIV disease[1-3]. To offer long term projections on clinical and economic implications to specific treatment strategies and to address the need to make clinical decisions where evidence from published studies is insufficient, costeffectiveness analyses offer strategic insights using model-based evaluations. Models used in cost-effectiveness analyses are often multidimensional and based on a large number of input parameters. In such model-based evaluations, efficacy estimates of drug regimens compared to placebo are critical for accurate delineation of alternative treatment strategies and cost-effectiveness comparisons. However, head-to-head placebo-controlled

\footnotetext{
* Correspondence: jchu6@partners.org

'Division of General Medicine, Department of Medicine, Massachusetts General Hospital, Boston, USA

Full list of author information is available at the end of the article
}

trials often are not feasible; they are expensive, time-consuming, and unethical if guidelines for a pharmaceutical intervention already exist [4]. Adjusted indirect comparison of randomized controlled trials has become an increasingly accepted method for assessing the effect of pharmaceutical interventions on survival outcomes, in the absence of placebo-controlled trials [5-8]. Within the framework of a cost-effectiveness model, often based on hundreds of parameters, it is not always feasible to use complex methods to derive every input parameter, especially for parameters not likely to affect major policy decisions.

Our goal was to illustrate a simple method for adjusting drug efficacy estimates according to differences in disease severity to derive parameters for a complex computer simulation model of HIV disease [1,9]. One study, for example, may compare regimen $\mathrm{A}$ to regimen $\mathrm{B}$, and another study may compare regimen $B$ to placebo. Adjusted indirect comparison provides a method for establishing the efficacy of regimen A compared to
C Biomed Central

() 2011 Chu et al; licensee BioMed Central Ltd. This is an Open Access article distributed under the terms of the Creative Commons Attribution License (http://creativecommons.org/licenses/by/2.0), which permits unrestricted use, distribution, and reproduction in any medium, provided the original work is properly cited. 
placebo, without losing the positive attributes of randomization.

Previous studies using adjusted indirect comparison estimated one-time probabilities and pooled the efficacies of drug regimens with varying doses $[5,7,10,11]$. Here, we establish a method for determining the efficacy of specific drug doses over time, thus allowing for predictions of treatment failure after any duration of therapy. We focused our illustration on prophylactic drugs for Mycobacterium avium complex (MAC) in patients infected with the human immunodeficiency virus (HIV) in the United States, because national guidelines recommend administering specific drugs and doses to prevent MAC [12]. Moreover, we also selected MAC as our illustration because of the availability of placebo-controlled trials for each guideline-recommended drug.

\section{Methods}

\section{Study selection}

We performed a MEDLINE search to identify randomized controlled trials of primary prophylaxis against MAC that were consistent with the current United States prophylaxis guidelines for HIV-infected patients[12]. We used the following search terms: Mycobacterium avium complex, randomized-controlled trial, placebo, rifabutin, azithromycin, and clarithromycin. We then reviewed the bibliographies of selected articles to identify other relevant studies. We considered data from randomized controlled clinical trials that reported follow-up time and administered primary prophylaxis for MAC, using one of the following drug regimens: $300 \mathrm{mg}$ rifabutin once daily, $1200 \mathrm{mg}$ azithromycin once weekly, or $500 \mathrm{mg}$ clarithromycin twice daily. These doses are based on the 2009 "Guidelines for Prevention and Treatment of Opportunistic Infections in HIVinfected Adults and Adolescents" [12]. To be included in this analysis, studies had to have at least two treatment arms and compare prophylactic regimens either to placebo directly, or to one another. Data on the number of subjects, follow-up time, and primary MAC events are included in Table 1. We collected additional data on mean CD4 count, number of patients on mono or dual antiretroviral therapy (ART), endpoint definitions, and inclusion or exclusion criteria from each study. For one pair of identically designed studies, we derived efficacy using the weighted averages of data from the two studies [13].

\section{Direct comparison}

If Trial 1 compared regimen A to placebo, we used Equation 1 to derive the efficacy of regimen A relative to placebo.

$$
\text { Efficacy } A=1-\frac{\text { Monthly Prob of failure } A}{\text { Monthly prob of failure Placebo }}
$$

We determined the two-sided 95\% confidence interval (CI) of each efficacy derived by direct comparison.

\section{Adjusted indirect comparison}

When direct comparison of a drug regimen to placebo was not possible, we made adjusted indirect comparisons. For example, when trial 1 compared regimen A to placebo, and trial 2 compared regimen $\mathrm{B}$ to regimen $\mathrm{A}$, we computed a "correction" factor to adjust for differences in baseline characteristic differences, including mean CD4 count and number of patients on ART, between the subjects of trial 1 and trial 2. The correction factor preserved the balance between the two randomized groups. Using Equation 2, we derived a correction factor to compare regimen A of trial 2 to regimen A of trial 1.

$$
\text { Correction Factor } A=\frac{\text { Monthly prob of failure } A, \text { trial2 }}{\text { Monthly prob of failure } A, \text { trial } 1}
$$

We then used Equation 3 to calculate the adjusted monthly probability of failure of regimen $\mathrm{B}$.

Adjusted monthly prob of failure $B=($ Correction factor $A) *($ Monthly Prob of failure $B)$

This adjusted monthly probability of failure allowed us to compare regimen $\mathrm{B}$ in trial 2 to placebo in trial 1 . We obtained the efficacy of regimen B using Equation 4.

$$
\text { Efficacy } B=1-\frac{\text { Adjusted monthly prob of failure } B}{\text { Monthly prob of failure Placebo }}
$$

We compared the direct and adjusted indirect efficacies of each regimen to assess the validity of adjusted indirect comparisons.

\section{Results}

\section{Characteristics of eligible trials}

We identified five eligible randomized controlled trials that included a total of 3,222 subjects (Table 1). Three studies compared one drug regimen to placebo, and two trials compared different prophylaxis regimens to each other.

\section{Efficacy by direct comparison}

We used data from three studies, by Nightingale et al., Pierce et al., and Oldfield et al. to compare rifabutin, clarithromycin, and azithromycin to placebo, directly (Table 2) [13-15]. The absolute efficacies of rifabutin, clarithromycin, and azithromycin, each compared to placebo, were estimated to be $53 \%$ (95\% CI, $48-61 \%), 66 \%$ (95\% CI, 61-74\%), and 66\% (95\% CI, 60-81\%).

\section{Efficacy by indirect comparison}

After adjusting the failure rate of rifabutin in the Benson et al. study to baseline characteristics in the Pierce et al. 
Table 1 Characteristics of 5 randomized controlled trials of primary prophylaxis against Mycobacterium avium complex in HIV-infected adults

\begin{tabular}{|c|c|c|c|c|c|c|c|c|}
\hline Study & Drug dose & $\begin{array}{c}\text { No. } \\
\text { subjects }\end{array}$ & 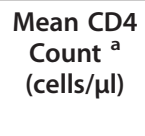 & $\begin{array}{l}\text { \% On } \\
\text { ART }\end{array}$ & $\begin{array}{l}\text { Median Follow- } \\
\text { up time }{ }^{b} \text { (days) }\end{array}$ & $\begin{array}{c}\text { Primary MAC } \\
\text { events }(N)\end{array}$ & $\begin{array}{l}\text { Direct monthly } \\
\text { failure rate } \\
(95 \% \mathrm{Cl})\end{array}$ & $\begin{array}{c}\text { Direct } \\
\text { monthly } \\
\text { probability } \\
\text { of failure }\end{array}$ \\
\hline \multirow[t]{2}{*}{$\begin{array}{l}\text { Nightingale 1993, study } \\
023 \text { and 027 [13] }\end{array}$} & $\begin{array}{l}\text { Rifabutin, } 300 \\
\text { mg, qd }\end{array}$ & 283 & 64 & 100 & $209^{d}$ & 24 & $0.012(0.007-0.017)$ & 0.012 \\
\hline & Placebo & 290 & 56 & 100 & $202^{d}$ & 51 & $0.027(0.019-0.0034)$ & 0.026 \\
\hline \multirow[t]{2}{*}{ Havlir 1996 [17] } & $\begin{array}{l}\text { Rifabutin, } 300 \\
\text { mg, qd }\end{array}$ & 223 & 47 & - & 514 & 52 & $0.014(0.010-0.018)$ & 0.014 \\
\hline & $\begin{array}{l}\text { Azithromycin, } \\
1200 \text { mg, qwk }\end{array}$ & 223 & 49 & - & 514 & 31 & $0.008(0.005-0.011)$ & 0.008 \\
\hline \multirow[t]{2}{*}{ Benson 2000 [16] } & $\begin{array}{l}\text { Rifabutin, } 300^{e} \\
\text { mg, qd }\end{array}$ & 391 & 30 & 75 & 574 & 59 & $0.008(0.006-0.010)$ & 0.008 \\
\hline & $\begin{array}{l}\text { Clarithromycin, } \\
500 \text { mg, bid }\end{array}$ & 398 & 27 & 73 & 595 & 36 & $0.005(0.003-0.006)$ & 0.005 \\
\hline \multirow[t]{2}{*}{ Pierce 1996 [14] } & $\begin{array}{l}\text { Clarithromycin, } \\
500 \text { mg, bid }\end{array}$ & 333 & 30 & - & $427^{f}$ & 19 & $0.004(0.002-0.006)$ & 0.004 \\
\hline & Placebo & 334 & 25 & - & $402^{f}$ & 53 & $0.012(0.009-0.015)$ & 0.012 \\
\hline \multirow[t]{2}{*}{ Oldfield 1998 [15] } & $\begin{array}{l}\text { Azithromycin, } \\
1200 \text { mg, qwk }\end{array}$ & 85 & 44 & - & $400^{d}$ & $9^{9}$ & $0.008(0.003-0.013)$ & 0.008 \\
\hline & Placebo & 89 & 44 & - & $340^{d}$ & $24^{9}$ & $0.024(0.015-0.034)$ & 0.024 \\
\hline
\end{tabular}

qd: once a day; bid: twice a day; qwk: once a week; MAC: Mycobacterium avium complex; ART: antiretroviral therapy; Cl: confidence interval

${ }^{\mathrm{a}}$ At baseline.

${ }^{\mathrm{b}}$ All patients on ART were on dual or mono therapy

'Study 023 and 027 are two identically designed studies. We calculated weighted averages the number of subjects, follow-up time, and number of new MAC events for the two studies.

${ }^{\mathrm{d} D u r a t i o n}$ on treatment

'This study was originally designed with a $450 \mathrm{mg}$ qd dosage but reduced to $300 \mathrm{mg}$ qd after 9 months.

${ }^{\mathrm{f}}$ Mean follow-up time

${ }^{9}$ The primary endpoints of this study were MAC symptoms and positive culture. We only included culture-positive events, to remain consistent with the other studies, which all used positive MAC cultures as primary endpoints.

study $[14,16]$, we estimated the adjusted indirect efficacy of rifabutin in Benson et al. to be $41 \%$. Similarly, we compared rifabutin in Havlir et al. to placebo in Oldfield et al., because both studies contained one azithromycin arm $[15,17]$. The efficacy of rifabutin in Havlir et al. was $44 \%$, compared to placebo in Oldfield et al.
When we adjusted the results of the Benson et al. study to baseline characteristics in Nightingale et al., using the rifabutin arms in each study, we estimated the efficacy of clarithromycin in Benson et al. compared to placebo in Nightingale et al. to be $73 \%[13,16]$. When we adjusted the results of the Havlir et al. study to

Table 2 Efficacy of MAC regimens by direct and adjusted indirect comparison

\begin{tabular}{|c|c|c|c|c|c|}
\hline Drug dose and study & $\begin{array}{l}\text { Method of efficacy } \\
\text { derivation }\end{array}$ & $\begin{array}{l}\text { Study used for } \\
\text { comparison }\end{array}$ & $\begin{array}{c}\text { Correction } \\
\text { factor }\end{array}$ & $\begin{array}{c}\text { Adjusted monthly probability } \\
\text { of failure }\end{array}$ & $\begin{array}{c}\text { \% Efficacy } \\
(95 \% \text { Cl) }\end{array}$ \\
\hline \multicolumn{6}{|l|}{ Rifabutin, $300 \mathrm{mg}$, qd } \\
\hline Nightingale 1993 [13] & Direct & - & - & - & $53(48-61)$ \\
\hline Havlir 1996 [17] & Adjusted indirect & Oldfield 1998 & $0.979^{\mathrm{a}}$ & 0.014 & 44 \\
\hline Benson 2000 [16] & Adjusted indirect & Pierce 1996 & $0.879^{b}$ & 0.007 & 41 \\
\hline \multicolumn{6}{|c|}{ Clarithromycin, 500 mg, bid } \\
\hline Pierce 1996 [14] & Direct & - & - & - & $66(61-74)$ \\
\hline Benson 2000 [16] & Adjusted indirect & Nightingale 1993 & $1.542^{c}$ & 0.007 & 73 \\
\hline \multicolumn{6}{|c|}{ Azithromycin, 1200 mg, qwk } \\
\hline Oldfield 1998 [15] & Direct & - & - & - & $66(60-81)$ \\
\hline Havlir 1996 [17] & Adjusted indirect & Nightingale 1993 & $0.896^{d}$ & 0.007 & 72 \\
\hline
\end{tabular}

Comparison of the monthly failure probabilities of:

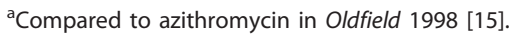

${ }^{\mathrm{b} C o m p a r e d}$ to clarithromycin in Pierce 1996 [14]

${ }^{c}$ Compared to rifabutin in Nightingale 1993 [13]

${ }^{\mathrm{d} C o m p a r e d}$ to rifabutin in Nightingale 1993[13] 
baseline characteristics in Nightingale et al. using the rifabutin arms in each study $[13,17]$, we estimated the efficacy of azithromycin in the Havlir et al. to be $72 \%$, compared to the Nightingale et al. placebo arm.

\section{Comparison of direct and adjusted indirect comparison methods}

The efficacies of clarithromycin and azithromycin derived by adjusted indirect comparison were not significantly different from the efficacies derived by direct comparison. However, our estimate of the efficacy of rifabutin by indirect comparison (41-44\%) was significantly lower than the efficacy derived by direct calculation (53\%).

\section{Discussion}

This paper illustrates a simple method that can be used to estimate input values for auxiliary parameters in multidimensional cost-effectiveness models. Since thorough methodological expertise in indirect comparisons may not always be accessible, the method illustrated in this paper could be used to derive efficacy of treatments where direct trials based on data are not readily available. To establish the efficacy of a drug regimen, it is necessary to compare outcomes for patients on and off therapy. While it is sometimes possible to derive this information directly from the results of randomized controlled trials, clinical trials are expected to provide enrolled participants with the best proven treatment, or at least the standard of care [4]. Thus, most studies compare different treatment options; studies that administer placebo to some subjects despite existing and accepted treatment options for the disease of interest lack equipoise and therefore are not ethical or feasible [4]. In this paper we have illustrated a simple method for indirectly estimating the dose-specific efficacy of drug regimens from reported results of randomized controlled trials without placebo arms by a straightforward adjustment for baseline clinical severity. When possible, we estimated the efficacies directly from the trials.

We found that the derived adjusted indirect efficacies of clarithromycin and azithromycin were similar to corresponding direct efficacies. However, the indirect efficacy of rifabutin was significantly lower than the efficacy derived by direct comparison. Unlike most other studies used in this analysis, the Nightingale et al. study reported mean duration on treatment, which is shorter than mean follow-up time. This substitution may therefore have led to an overestimation of the direct efficacy of rifabutin. The greater efficacy of rifabutin in the direct comparison may also be attributed to the greater proportion of patients on ART in this trial.

Our proposed method was consistent with that of previous studies showing that adjusted indirect comparison reduces bias in drug efficacy calculations [5-7,11]. Our inclusion criteria were stricter than those in previous studies, because we examined outcomes only from trials that compared drug regimens with specific doses and that provided results at several time points. Thus, we avoided having to pool results from various doses of the same drug regimen. Our results may be more accurate than previous studies for the specific doses examined, since we only included trials that administered the doses recommended in the United States "Guidelines for Prevention and Treatment of Opportunistic Infections in HIV-Infected Adults and Adolescents"[12]. Similar results may be obtained using Indirect Treatment Comparisons (ITC) Software from the Canadian Agency for Drugs and Health Technologies [18]. While this offers a means of validation of the methods in this paper, a step-by-step description may be useful to those who do not have direct access to the ITC software, or for further understanding of the insights provided.

One of the main purposes of the indirect comparisons is to make stronger inferences about comparisons being studied. We recognize the scarcity of placebo-controlled trials in the HIV/AIDS field, particularly among newer trials, and we believe that using older placebo-controlled trials, as we have done in our illustration, for the purpose of adjusted indirect comparisons, is acceptable. Our study was limited by the number of studies that could be used to derive OI prophylaxis efficacy. Only five studies met the inclusion criteria. However, because the focus of this analysis was to illustrate simple and replicable methodology for adjusted indirect comparison of drug regimens, the small number of included studies does not deter from this goal. Moreover, two studies did not report follow-up time $[13,15]$. For these studies, we calculated efficacy by substituting follow-up time with mean duration on treatment to calculate efficacy. The Oldfield et al. study was terminated early because administering placebo became inappropriate when the results of a separate azithromycin efficacy trial [15]. It may be reasonable to assume that most patients were on treatment at the time of study discontinuation, and thus that the unreported mean follow-up time is very similar to the mean duration on treatment. However, treatment duration in the Nightingale et al. study may have been greater than the true unreported mean follow-up time, and could have led to an overestimation of the efficacy of rifabutin. While this method offers a useful approach for derivation of point estimates, an extensive set of sensitivity analyses are necessary to examine the robustness of policy conclusions to uncertainty in parameter values. If a parameter is influential, more sophisticated methods should be employed to obtain a more precise value of parameter.

The prevalence of MAC and other opportunistic infections among HIV-infected patients in the United States 
and Europe has greatly decreased since the earlier years of the HIV epidemic, due to the success of combination antiretroviral therapy [19]. However, methods presented in this study continue to be applicable to resourcelimited settings, where the use of opportunistic infection prophylaxis in the absence of ART is still widespread. In these areas, the WHO recommends lifelong prophylaxis for fungal and bacterial infections, as well as for Pneumocystis carinii Pneumonia with drugs such as fluconazole and cotrimoxazole [20]. In the United States, recommendations for the prevention of opportunistic infections continue to be revised regularly in the national guidelines $[12,21,22]$. Similar indirect comparison methods may be useful in comparing effective firstline antiretroviral regimens in the United States and in many countries-such as those containing efavirenz, darunavir, atazanavir, and raltegravir in the United Statesthat have not been compared directly with each other [23-25]. These methods can also be used to compare second-line or subsequent ART regimens when efficacy data have been published but direct comparisons may have not been done.

\section{Conclusion}

The methodology demonstrated in this study is applicable to policy and implementation research, for which it is necessary to know the absolute efficacy of specific doses of pharmaceutical interventions as compared to no intervention, to predict the outcomes of treatment policies. As treatment options for HIV disease, both in terms of opportunistic infection prophylaxis and ART, continue to grow, these methods can help estimate efficacies across a wide range of available and useful therapeutic regimens.

\section{Acknowledgements}

This study was supported by the National Institute of Allergy and Infectious Diseases (R37 Al042006, R01 Al058736, K24 Al062476).

\section{Author details \\ 'Division of General Medicine, Department of Medicine, Massachusetts General Hospital, Boston, USA. ${ }^{2}$ Division of Infectious Disease, Department of Medicine, Massachusetts General Hospital, Boston, USA. ${ }^{3}$ The Harvard University Center for AIDS Research, Harvard Medical School, Boston, USA. ${ }^{4}$ Department of Orthopaedic Surgery, Brigham and Women's Hospital, Boston, USA. ${ }^{5}$ Faculté de Médecine de Lille, Centre Hospitalier de Tourcoing, Tourcoing, France.}

\section{Authors' contributions}

JC, CS, and EL conceived and designed the study. JC and CS drafted the manuscript. $K F, Y Y$, and EL provided critical revisions of the article for important intellectual content. All authors read and approved the final manuscript.

\section{Competing interests}

The authors declare that they have no competing interests.

Received: 3 October 2010 Accepted: 10 March 2011 Published: 10 March 2011

\section{References}

1. Goldie SJ, et al: Cost-effectiveness of HIV treatment in resource-poor settings-the case of Cote d'Ivoire. N Engl J Med 2006, 355(11):1141-53.

2. Sanders GD, et al: Cost-effectiveness of screening for HIV in the era of highly active antiretroviral therapy. N Engl J Med 2005, 352(6):570-85.

3. Walensky RP, et al: When to start antiretroviral therapy in resourcelimited settings. Ann Intern Med 2009, 151(3):157-66.

4. Rothman KJ, Michels KB: The continuing unethical use of placebo controls. N Engl J Med 1994, 331(6):394-8.

5. Bucher $\mathrm{HC}$, et al: The results of direct and indirect treatment comparisons in meta-analysis of randomized controlled trials. J Clin Epidemiol 1997, 50(6):683-91.

6. Song F, Glenny AM, Altman DG: Indirect comparison in evaluating relative efficacy illustrated by antimicrobial prophylaxis in colorectal surgery. Control Clin Trials 2000, 21(5):488-97.

7. Fisher LD, Gent M, Buller HR: Active-control trials: how would a new agent compare with placebo? A method illustrated with clopidogrel, aspirin, and placebo. Am Heart J 2001, 141(1):26-32.

8. Yazdanpanah $Y$, et al: Clinical efficacy of antiretroviral combination therapy based on protease inhibitors or non-nucleoside analogue reverse transcriptase inhibitors: indirect comparison of controlled trials. BMJ 2004, 328(7434):249.

9. Paltiel $A D$, et al: Expanded screening for HIV in the United States-an analysis of cost-effectiveness. N Engl J Med 2005, 352(6):586-95.

10. Song F, et al: Validity of indirect comparison for estimating efficacy of competing interventions: empirical evidence from published metaanalyses. BMJ 2003, 326(7387):472.

11. Song F, Harvey I, Lilford R: Adjusted indirect comparison may be less biased than direct comparison for evaluating new pharmaceutical interventions. J Clin Epidemiol 2008, 61(5):455-63.

12. CDC: Guidelines for Prevention and Treatment of Opportunistic Infections in HIV-infected Adults and Adolescents. Recommendations from the CDC, the National Institues of Health, and the HIV Medicine Association of the Infectious Diseases Society of America. MMWR 2009, 50(RR-4):1-207.

13. Nightingale SD, et al: Two controlled trials of rifabutin prophylaxis against Mycobacterium avium complex infection in AIDS. $N$ Engl J Med 1993, 329(12):828-33.

14. Pierce $M$, et al: A randomized trial of clarithromycin as prophylaxis against disseminated Mycobacterium avium complex infection in patients with advanced acquired immunodeficiency syndrome. $N$ Engl J Med 1996, 335(6):384-91.

15. Oldfield EC, et al: Once weekly azithromycin therapy for prevention of Mycobacterium avium complex infection in patients with AIDS: a randomized, double-blind, placebo-controlled multicenter trial. Clin Infect Dis 1998, 26(3):611-9.

16. Benson CA, et al: Clarithromycin or rifabutin alone or in combination for primary prophylaxis of Mycobacterium avium complex disease in patients with AIDS: A randomized, double-blind, placebo-controlled trial. The AIDS Clinical Trials Group 196/Terry Beirn Community Programs for Clinical Research on AIDS 009 Protocol Team. J Infect Dis 2000, 181(4):1289-97.

17. Havlir DV, et al: Prophylaxis against disseminated Mycobacterium avium complex with weekly azithromycin, daily rifabutin, or both. California Collaborative Treatment Group. N Engl J Med 1996, 335(6):392-8.

18. Wells $\mathrm{G}$, et al: Indirect treatment comparison [computer program]. Canadian Agency for Drugs and Technologies in Health: Ottawa; 2009.

19. Furrer $H$, et al: Discontinuing or withholding primary prophylaxis against Mycobacterium avium in patients on successful antiretroviral combination therapy. The Swiss HIV Cohort Study. AIDS 2000, 14(10):1409-12.

20. Essential prevention and care interventions for adults and adolescents living with HIV in resource-limited settings. World Health Organization: Geneva; 2008.

21. CDC: Guidelines for Preventing Opportunistic Infections Among HIVInfected Persons-2002. Recommendations of the U.S. Public Health Service and the Infectious Diseases Society of America. MMWR 2002, 51(RR-8):1-46.

22. CDC: Treating Opportunistic Infections Among HIV-Infected Adults and Adolescents: recommendations from CDC. MMWR 2004, 51(RR-15):1-112. 
23. van Leth $F$, et al: Comparison of first-line antiretroviral therapy with regimens including nevirapine, efavirenz, or both drugs, plus stavudine and lamivudine: a randomised open-label trial, the 2NN Study. Lancet 2004, 363(9417):1253-63.

24. Squires K, et al: Comparison of once-daily atazanavir with efavirenz, each in combination with fixed-dose zidovudine and lamivudine, as initial therapy for patients infected with HIV. J Acquir Immune Defic Syndr 2004, 36(5):1011-9.

25. ClinicalTrials.gov [Internet]. Bethesda (MD): National Institute of Allergy and Infectious Diseases. 2008 Dec 18. Identifier NCT00211954, Comparative Study of Three NNRTI-Sparing HAART Regimens; 2010 Apr 2010 [cited 2010 Jul 08]; [about 6 screens]. [http://clinicaltrials.gov/ct2/ show/NCT00811954?term=ACTG+5257\&rank=3].

doi:10.1186/1742-6405-8-14

Cite this article as: Chu et al:: Drug efficacy by direct and adjusted indirect comparison to placebo: An illustration by Mycobacterium avium complex prophylaxis in HIV. AIDS Research and Therapy 2011 8:14.

\section{Submit your next manuscript to BioMed Central} and take full advantage of:

- Convenient online submission

- Thorough peer review

- No space constraints or color figure charges

- Immediate publication on acceptance

- Inclusion in PubMed, CAS, Scopus and Google Scholar

- Research which is freely available for redistribution

Submit your manuscript at www.biomedcentral.com/submit 